\title{
Development and Validation of Brain Metastasis Detection System using Artificial Neural Network on MR
}

\author{
Young Jae Kimª, Kwang Gi Kimª, \\ aDepartment of Biomedical Engineering, Gachon University, 21, Namdong-daero, 774 beon-gil, Namdong-gu, \\ Incheon(21565), Korea \\ *Corresponding Author: kimkg@gachon.ac.kr
}

\begin{abstract}
Brain tumor detection and segmentation in magnetic resonance images is important in medical diagnosis because it provides information associated to anatomical structures as well as potential abnormal tissues necessary to treatment planning and patient follow-up. In this research, we proposed the fully automated brain metastasis detection system. We performed normalization and brain region extraction in preprocessing. We developed a CAD system for brain metastasis detection using template matching and $\mathrm{K}$-means clustering algorithms for candidate detection, and artificial neural network for false-positive reduction. The proposed method showed a sensitivity of $87.3 \%$ (117/134 nodules). In the future, we expected that further research would improve the performance of the proposed method.
\end{abstract}

Keywords: Brain metastasis, MRI, Computer-aided diagnosis, Artificial neural network, detection.

\section{Introduction}

In the brain, the most common tumor is the metastatic brain tumor. If the brain metastasis is not treated, the prognosis is very bad ${ }^{(1)}$. Whole-brain radiation therapy (WBRT) has long been the primary treatment for brain metastases ${ }^{(2,3)}$. However, WBRT may induce neurocognitive function impairment in some patients. Therefore, patients with less than 10 brain metastasis are using stereotactic radiosurgery as their primary treatment ${ }^{(4)}$. In this regard, accurate detection of number and location of brain metastases on magnetic resonance (MR) has become crucial in order to choose the most appropriate treatment method.

Recently, many researchers are studying computeraided diagnosis $(\mathrm{CAD})$ methods of various lesions to provide a second opinion for radiologists. Previous studies have shown that CAD can help increase sensitivity to lesions in the breast, lung, and colon ${ }^{(5-7)}$.

In this paper, we developed a brain metastases detection software using the CAD technology, and we aimed to assess the effect of CAD software on radiologists' diagnostic performance.

\section{Material}

For our study, we collected MR images from 110 patients with brain metastases at Seoul National University Bundang Hospital. In the collected data, 80 patients (450 nodules) were designated as the training set and 30 patients (134 nodules) were designated as the test set. The size of the nodules ranged from $1 \mathrm{~mm}$ to $9 \mathrm{~mm}$.

MR images were obtained with a $1.5 \mathrm{~T}$ (Intera; Philips Healthcare, Best, the Netherlands) or a 3.0 T (Acheiva or Ingenia; Philips Healthcare, Best, the Netherlands) MR scanner. The image had 520 X 520 resolution, $0.46875 \mathrm{~mm}$ pixel spacing, and $1.0 \mathrm{~mm}$ slice thickness. Information about the patient was deleted and all data were classified and managed using serial numbers.

Programs used in the experiment were Microsoft Visual Studio (Ver. 2010, Microsoft, Redmond, WA, USA), ITK (Insight Segmentation and Registration Toolkit, Kitware Inc., NY, USA), and VTK (Visualization Toolkit, Kitware Inc., NY, USA).

\section{Method}

The algorithms of the developed CAD software consists of normalization phase, brain segmentation phase, brain metastasis detection phase, false positive reduction 
phase. Fig 1 shows the complete flowchart of the proposed algorithm.

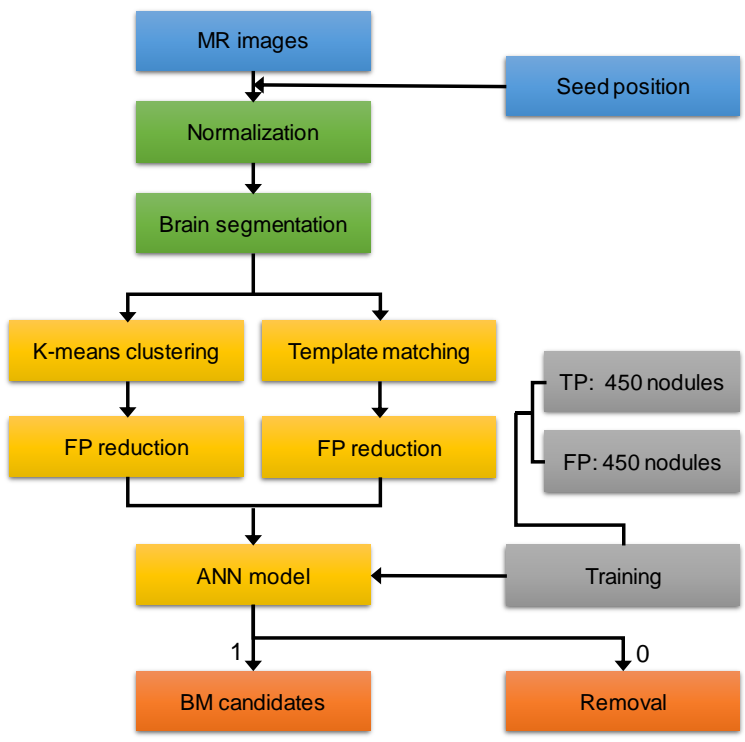

Fig. 1. Flowchart of proposed method.

\subsection{Normalization}

The attenuation value of Computed Tomography (CT) uses an absolute value, but the signal intensity of MR imaging is a relative value. Thus, the range of signal intensity depends on the scanning parameters on MR.

To solve this problem, we normalized the image by resampling the signal from the entire image to the same range based on the signal strength of the manually selected initial seed position in the gray matter.

\subsection{Brain segmentation}

We tried to limit the region of interest in the brain region by extracting the brain from the MR images. Limiting the algorithm to the brain region can reduce the potential for false positive (FP) brain metastasis in the anatomical structure outside the brain region.

We used the 3D seeded spherical-based region growing (SSRG) algorithm for brain segmentation based on the manually determined seed position in the gray matter. Seed region growing (SRG) algorithm is a common method of three-dimensionally expanding seed regions $(\mathrm{x}, \mathrm{y}, \mathrm{z})$ to separate homogeneous regions ${ }^{(8)}$. The SRG algorithm expands the region by pixel. Thus, if the signal intensity of the brain region is similar to that of other adjacent structures, there is a possibility that brain segmentation will fail with just one pixel. To solve this problem, we have developed an
SSRG algorithm that extends the area when all the pixels in the region conform to the expansion conditions. Thus, it is possible to prevent the region from expanding to another adjacent structure having a similar signal strength.

\subsection{Brain metastasis detection}

Brain metastasis are well-defined borders with surrounding anatomical tissue ${ }^{(9)}$. Brain metastasis typically has a spheroid like structure and contrast is enhanced at T1WI. However, some large BM has an irregular structure. In addition, the attenuation is low when internal necrosis. We proposed two kinds of algorithms according to the size of the nodules based on the characteristics of brain metastasis morphology.

We used a three-dimensional template-matching algorithm for brain metastasis detection with a small size spheroid like structure. Specifically, we used two spherical template models with a solid type and an inner-hole type to compensate for the internal necrosis. Three size templates were created for each of the two models, with diameters of 2 $\mathrm{mm}, 3 \mathrm{~mm}$, and $4 \mathrm{~mm}$. Moreover, size of the inner hole was determined to be $50 \%$ of the each template size. Within the extracted brain volume, we performed a convolution on the brain volume by template models. We detected BM candidates with evaluating the similarity in each position in

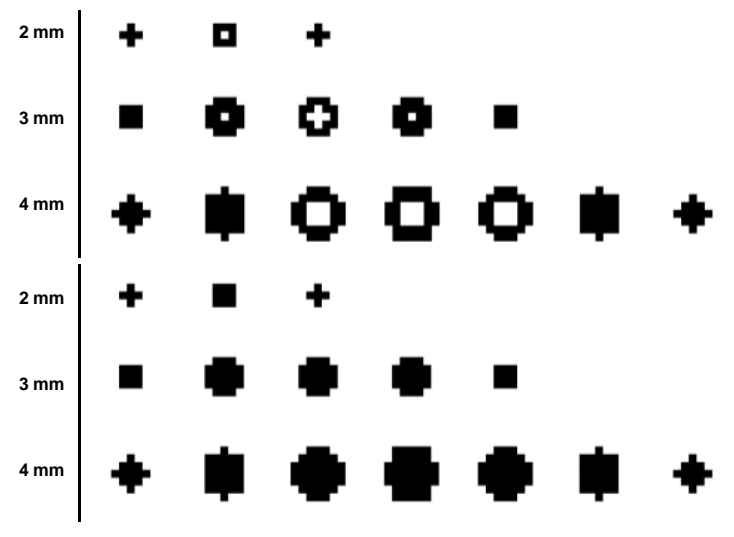

Fig. 2. Examples of six templates.

the brain volume.

We used a K-means clustering algorithm for brain metastasis detection with a large size irregular structure. Kmeans clustering is one of the unsupervised classification techniques. The K-means clustering is an algorithm for grouping data onto $\mathrm{k}$ clusters. The data are distributed over the nearest cluster by calculating the Euclidean distance between the data and the center of each cluster ${ }^{(10)}$. We defined seven clusters then performed the K-means 
clustering on the attenuation of all coordinate in the brain images. Moreover, we aligned each cluster of a mean of attenuation. On the aligned clusters, both ends have the highest or lowest attenuation. In other words, there is a high probability that clusters at both ends are enhanced brain metastasis or brain metastasis including necrotic tissue. We performed 3D labeling on the coordinates of clusters at both ends. Morphological features were calculated for each label and used for discrimination of brain metastasis. Finally, the labels with the feature values greater than the experimentally defined thresholds were considered potential candidates.

\subsection{False positive reduction using machine learning}

We removed the false positives by discriminating brain metastasis candidates to improve the accuracy. For the discrimination of the nodule candidates, we used the artificial neural network (ANN) algorithm that is one of the machine learning techniques. ANN is a mathematical model based on biological neural networks ${ }^{(11)}$. It consists of an interconnected group of the artificial neurons and neurons are organized into layers. We used three layers with input, output and hidden layer. The input layer consists of 30 neurons, and we used 30 features measured from the BM candidate images as input neurons.

We measured 272 features of based on histogram, morphology, and texture to select 30 input features. And significant features were selected by logistic regression analysis. Finally, based on the selected features by logistic regression analysis, we selected 30 features based on empirical judgement. The features used are as follows: volume, min, max, mean, standard deviation, variance, skewness, kurtosis, energy, entropy, fractal dimension(box counting), GLCM-contrast, GLCM-dissimilarity, GLCMhomogeneity, GLCM-ASM, GLCM-energy, GLCMprobability max, GLCM-entropy, GLCM-correlation, GLCM-mean reference, GLCM-mean neighbor, GLCMvariance reference, GLCM-variance neighbor, GLCMstandard deviation reference, GLCM-standard deviation neighbor, GLRLM-LRE, GLRLM-GLN, GLRLM-RLN, GLRLM-LGRE, GLRLM-HGRE.

The output layer consists of two neurons representing $\mathrm{BM}$ and non-BM. The ANN model used in this study was trained using a backpropagation algorithm with a feedforward architecture and hyperbolic tangent activation. The result of the output node indicates the likelihood that the nodule will be classified into its respective class. Thus, in this study, the output was interpreted as the probability that a brain metastasis candidate is a true-positive nodule.

\section{Result}

We detected the brain metastasis using a template matching and K-means clustering algorithm. Moreover, we reduced FP by using ANN algorithm that is a machine learning technique. The result is shown in Figure 4.

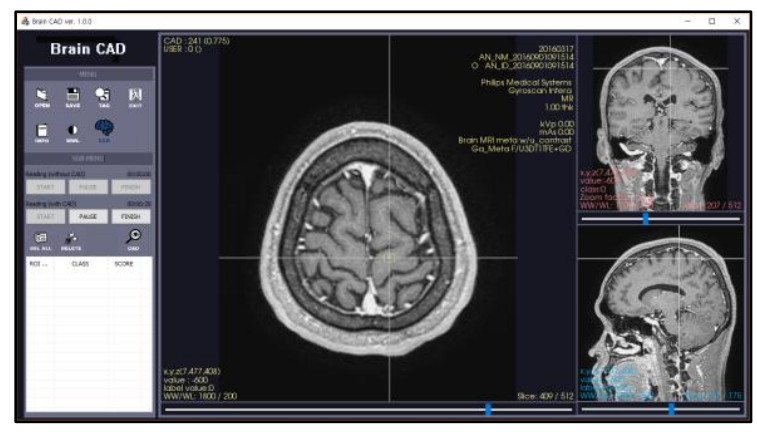

Fig. 3. Brain metastasis detection software.

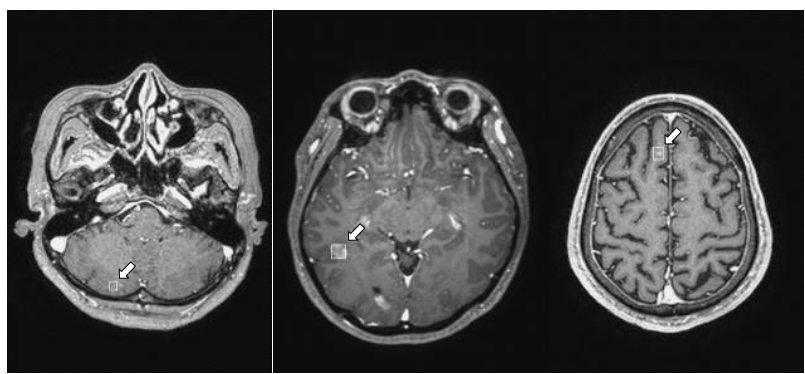

Fig. 4. Examples of brain metastasis detection results.

The proposed method showed a sensitivity of $87.3 \%$ $(117 / 134$ nodules). When tiny nodules of less than or equal to $2 \mathrm{~mm}$ in diameter were excluded, sensitivity was increased to $92.7 \%$ (89/96 nodules). In addition, the proposed method showed an average processing time of $264.7 \mathrm{~s}$.

Table 1. Results of performance for brain metastasis detection algorithm.

\begin{tabular}{|c|c|c|}
\hline & $\begin{array}{c}\text { Brain Metastasis } \\
(\geq 1 \mathrm{~mm})\end{array}$ & $\begin{array}{c}\text { Brain Metastasis } \\
(\geq 2 \mathrm{~mm})\end{array}$ \\
\hline Sensitivity & $87.3 \%$ & $92.7 \%$ \\
& $(117 / 134)$ & $(89 / 96)$ \\
\hline
\end{tabular}

The average sensitivity and false positive rate (FPR) per patient of brain metastasis detection without CAD for the four reviewers were $77.6 \%(66.4 \% \sim 88.1 \%)$ and $0.18(0.05$ $\sim 0.25)$, respectively. With CAD, the sensitivity and FPR per patient changed to $81.9 \%(75.3 \% \sim 88.8 \%)$ and $0.18(0.07$ $\sim 0.3$ ), respectively. 


\section{Discussion}

In the present study, we developed a CAD software and evaluated its stand-alone performance, and then conducted an observer performance study. Notably, the two trainees detected 22 additional TP nodules after reviewing the CAD results. While CAD significantly improved the overall performance of the reviewers, statistically significant improvement was noted only for less-experienced reviewers. As a result, significant effect of time reduction and quantitative as well as objective results can be achieved when our proposed method for brain metastasis.

The strategy of our proposed algorithm was to first detect the BM candidates as sensitive as possible, and then discriminate TP nodules from FP nodules. We used a template matching algorithm to find a small size BM. While other similar studies used larger templates with a minimum diameter of $3.4 \mathrm{~mm}$, we were able to find smaller nodules by using smaller templates. In addition, other studies used only one type of template model ${ }^{(12,13)}$, whereas we used two spherical types of template models (a solid and an innerhole). Thus, we were able to detect small necrotic nodules accurately.

However, we were limited in reducing FP despite using machine learning, We believe the performance could be improved using larger amount of data and using more algorithms such as deep learning. If additional studies are complementary to CAD software, the CAD as a second reader will help improve diagnostic performance, especially when radiologists find $\mathrm{BM}$ in $\mathrm{MR}$ imaging.

\section{Acknowledgment}

This work was supported by a commissioned research grant from the Electronics and Telecommunications Research Institute (grant no. 1500-2017-0001).

\section{References}

(1) Richards $\mathrm{P}$, and Mckissock W. : "Intracranial Metastases”, Br Med J., Vol. 1, No. 5322, pp. 15-18, 1963

(2) Chao JH, Phillips R, Nickson JJ. : "Roentgen-ray therapy of cerebral metastases", Cancer, Vol. 7, No. 4, pp. 682-689, 1954

(3) Chang EL, Wefel JS, Hess KR, Allen PK, et al. : "Neurocognition in patients with brain metastases treated with radiosurgery or radiosurgery plus whole- brain irradiation: a randomised controlled trial", Lancet Oncol., Vol. 10, No. 11, pp. 1037-1044, 2009

(4) Chang WS, Kim HY, Chang JW, Park YG, Chang JH. : "Analysis of radiosurgical results in patients with brain metastases according to the number of brain lesions: is stereotactic radiosurgery effective for multiple brain metastases?", J Neurosurg., Vol. 113, No. Special Suppl, pp. 73-78, 2010

(5) Chan H-P, Kunio D, Vybrony CJ, Schmidt RA, Metz CE, Lam KL, et al. : "Improvement in radiologists' detection of clustered microcalcifications on mammograms: the potential of computer-aided diagnosis", Invest Radiol., Vol. 25, No. 10, pp. 1102-1110, 1990

(6) Kobayashi T, Xu X-W, MacMahon H, Metz CE, Doi K. : "Effect of a computer-aided diagnosis scheme on radiologists' performance in detection of lung nodules on radiograph", Radiology, Vol. 199, No. 3, pp. 843-848, 1996

(7) Baker ME, Bogoni L, Obuchowski NA, et al. : "Computer-aided detection of colorectal polyps : can it improve sensitivity of less-experienced readers? preliminary findings1, Radiology, Vol. 245, No. 1, pp. 140-149, 2007

(8) Revol-Muller C, Peyrin F, Carrillon Y, Odet C. : "Automated 3D region growing algorithm based on an assessment function", Pattern Recognit Lett., Vol. 23, No. 1, pp. 137-150, 2002

(9) Jagannathan J, Sherman JH, Mehta GU, Chin LS. : "Radiobiology of brain metastasis: applications in stereotactic radiosurgery", Neurosurg Focus., Vol. 22, No. 3, pp. E4, 2007

(10) Juang L-HH, Wu M-NN. : "MRI brain lesion image detection based on color-converted K-means clustering segmentation", Measurement, Vol. 43, No. 7, pp. 941949,2010

(11) Bollschweiler EH, Mönig SP, Hensler K, Baldus SE, et al., : "Artificial neural network for prediction of lymph node metastases in gastric cancer: a phase II diagnostic study, Ann Surg Oncol., Vol. 11, No. 5, pp. 506-511, 2004

(12) Ambrosini RD, Wang P, O’Dell WG. : "Computer-aided detection of metastatic brain tumors using automated three-dimensional template matching", J Magn Reson Imaging, Vol. 31, No. 1, pp. 85-93, 2010

(13) Pérez-Ramírez Ú, Arana E, Moratal D. : "Brain metastases detection on MR by means of threedimensional tumor-appearance template matching", J Magn Reson Imaging, Vol. 44, No. 3, pp. 1-11, 2016 